\title{
Surgical versus conservative treatment following acute rupture of the Achilles tendon: is there a pedobarographic difference?
}

This article was published in the following Dove Press journal:

Therapeutics and Clinical Risk Management

29 August 2016

Number of times this article has been viewed

\author{
Fatih Karaaslan' \\ Musa Uğur Mermerkaya' \\ Alper Çıraklı² \\ Sinan Karaoğlu ${ }^{3}$ \\ Fuat Duygulu² \\ 'Department of Orthopaedics and \\ Traumatology, Faculty of Medicine, \\ Bozok University, Yozgat, Turkey; \\ ${ }^{2}$ Department of Orthopaedics and \\ Traumatology, Kayseri Training \\ Hospital, Kayseri, Turkey; ${ }^{3}$ Department \\ of Orthopaedics and Traumatology, \\ Memorial Kayseri Hospital, \\ Kayseri, Turkey
}

Introduction: Controversy remains regarding the optimal treatment method and postoperative rehabilitation of acute Achilles tendon ruptures. In this study, pedobarographic assessments of surgical and conservative treatments were compared.

Material and methods: A prospective assessment was made of 16 patients (eight surgical, eight conservative) and eight healthy controls using a plantar pressure measurement system. Biomechanical gait parameters were obtained using the Footscan dynamic gait analysis system. Kruskal-Wallis and Mann-Whitney $U$-tests were used for the evaluation of data.

Results: Nineteen males and five females were assessed, with an average age of $42.0 \pm 11.9$ years. Follow-up was completed in 16 patients. No statistically significant difference was determined between the two treatment groups with regard to the gait analysis, but a difference was observed with the control group $(P<0.001)$. All patients were able to resume their prior activities after 6 months and regained normal ranges of motion, with a high rate of satisfaction. Most of the patients $(75 \%)$ were able to return to their pre-injury level of activities.

Conclusion: Satisfactory results were obtained through conservative treatment of acute ruptures of the Achilles tendon. No significant differences or complications were observed in the group managed conservatively versus the group treated surgically. Further studies including 3D gait analyses and tendon biomechanical research are required to further investigate this issue.

Keywords: Achilles tendon, acute rupture, pedobarographic analysis

\section{Introduction}

Ruptures of the Achilles tendon generally occur during sports activities and are seen at higher rates in males than females. The incidence has been reported as $18 / 100,000 .{ }^{1-3}$ The increasing popularity of recreational activities has contributed to a rise in this injury.

Regarding the knee, many studies have verified an increased incidence of ligamentous injuries and a congruently increased number of surgeries for female athletes compared with male athletes. Nevertheless, the incidence of Achilles tendon rupture is lower in females than in males, with a ratio ranging between $1.67 / 1$ and 6.90/1. ${ }^{4-6}$

Treatment management has continued to develop over the past 20 years. There are many studies in the literature related to the treatment for Achilles tendon tears. However, optimal treatment methods and postoperative rehabilitation programs are still part of a debate. ${ }^{1,7,8}$ Although various studies have shown faster returns to pre-injury activity levels after surgical treatment in athletes, there is currently no gold standard for the treatment of Achilles tendon ruptures. ${ }^{9}$

Studies that have compared different treatment methods have focused mostly on complications and results. Rehabilitation after rupture of the Achilles tendon is slow
Correspondence: Fatih Karaaslan

Department of Orthopedics and Traumatology, Faculty of Medicine, Bozok University, 18 Madrasa Adnan Menderes, Boulevard, Yozgat 66200, Turkey

Tel +903542127060

Email fkaraaslan@gmail.com 
and it may take longer than 3 months. This is more important for professional athletes. Despite most of the patients reporting good long-term results, a significant number of patients experience persistent symptoms for years. ${ }^{1,10}$ A key factor limiting recovery is abnormal gait. ${ }^{10,11}$ Measurement of plantar pressure is an important step of any gait analysis.

The aim of this study was to compare pedobarographic assessment results of cases treated surgically and conservatively with control subjects. A secondary question was whether there was any difference between treatment modalities.

\section{Material and methods}

This study used an observational, prospective design and protocol was approved by the Bozok University Faculty of Medicine Ethics Committee. Patients were included in the study if they had suffered a mid-body rupture of the Achilles tendon during sports activity and were treated with open surgery or conservative treatment. Diabetes mellitus, previous Achilles tendon rupture, other lower leg injuries, immunosuppressive therapy, and neurovascular diseases were defined as exclusion criteria. If the rupture occurred more than 10 days prior, was not due to trauma, or occurred outside of sports or physical activity, patients were also excluded.

In total, 16 patients (eight surgical, eight conservative) and eight healthy control subjects were assessed using a plantar pressure measurement system with an average follow-up of 10 months. Written informed consent was obtained from the patients. The $225 \times 445 \mathrm{~mm}$ sensitive monitors of the apparatus were mounted onto a $1 \times 7 \mathrm{~m}$ walking platform. Both feet of each patient were analyzed three times. The data obtained from the normal feet were taken as the control group. Dynamic and static pressure maps of the feet were taken using platforms that measured the floor reaction strength and pressure variations in the foot, heel, and forefoot during the walking phase.

The F-scan system (Tekscan Inc., Boston, MA, USA) uses thin, in-shoe pressure pads to record plantar pressures (Figure 1). The pressure pads have four sensors per $\mathrm{cm}^{2}$ and a sampling frequency of $500 \mathrm{~Hz}$. The patients were then requested to walk along a straight line on a flat surface at a normal walking pace. Ten cycles of gait were recorded and the data from the fourth, fifth, and sixth cycles were used in the analysis. Because of the tendon's biomechanical property, plantar flexion torque of the gastrocnemius and soleus muscles was transmitted by tendon, ${ }^{12}$ the main focus of the analysis was the terminal stance and pre-swing phases of the gait cycle. During these phases active muscle contractions occur. In the terminal stance phase the heel of the reference foot rises while the toe is still in contact with the ground while the patient's

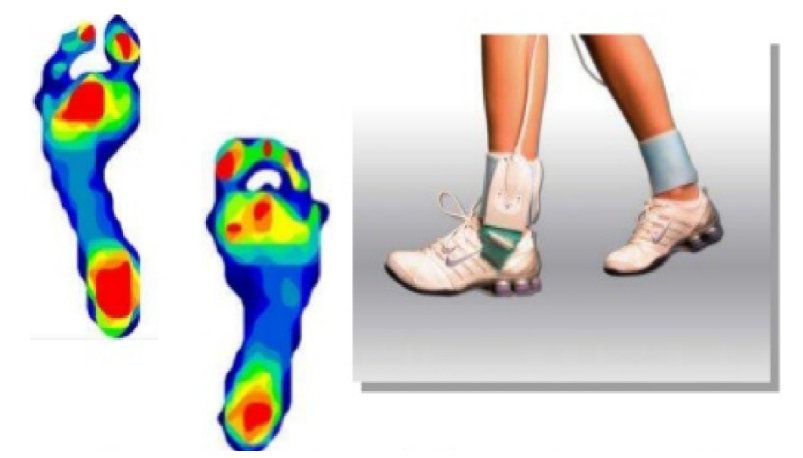

Figure I The F-scan system.

center of gravity is over the center of the foot. During the preswing phase the toe of the reference foot rises and swings in air. Regarding these phases the following two variables were assessed: 1) duration of the terminal stance and pre-swing phases; 2) the mean and maximum peak plantar pressures. Each measurement is given as a percentage of the value obtained from the control group. Therefore, the assessment is directly related to the difference between the injured limb and uninjured control group. ${ }^{10}$ Biomechanical gait parameters were obtained using the Footscan dynamic gait analysis system. Following acute rupture of the Achilles tendon, surgical repair with an open technique was used in eight patients, with eight others undergoing conservative treatment.

\section{Surgical technique}

All procedures were performed under spinal anesthesia with the patient in a prone position. A thigh pneumatic tourniquet (350 $\mathrm{mmHg}$ ) was used. The approach was medial to the midline of the tendon. The tendon sheath was dissected. The ruptured tendon was repaired in a Kessler pattern using a slowly absorbable suture. The tendon sheath was then closed, along with the skin, using a water-tight, but non-ischemic, suture pattern. No drain was used in any case. All patients were immobilized with a below-knee splint, with the ankle in a $30^{\circ}$ plantar flexion. Patients were discharged on the first day after the operation.

\section{Conservative technique}

Patients were treated immediately with platelet-rich plasma (PRP) application before VACOped bracing (OPED GmbH, Valley/Oberlaindern, Germany), with the foot in the equinus position. PRP preparation consisted of centrifuging a $60 \mathrm{~mL}$ venous blood sample of the patient, from which $6 \mathrm{~mL}$ of plasma containing concentrated platelets, white blood cells, and growth factors were obtained. Then, $\sim 4 \mathrm{~mL}$ of PRP was injected into the tendon fibers. The initial VACOped was in a full equinus position for 4 weeks; it was then changed to a 
semi-equinus cast for 4 weeks, followed by a VACOped in a neutral position for 2 weeks.

\section{Postoperative regimen and analysis methods}

Patients were advised to avoid weight-bearing while in the equinus and semi-equinus orthesis, and partial weightbearing was then allowed in the neutral position. In the first 2 weeks, they were asked to concentrate on increasing their range of motion and non-weight-bearing dorsiflexion stretches were permitted. Patients were encouraged to commence weight-bearing on removal of the brace. From weeks 2 to 6 , ankle range of movement was emphasized using non-weight-bearing range-of-movement exercises and weight-bearing stretches. Strengthening of the calf muscle was started using bilateral heel raises and eccentric calf loading, before progressing to unilateral heel raises as strength in the injured limb recovered. Proprioception exercises were used, concentrating on single-leg balance. During this period, patients were seen twice weekly by the supervising physical therapist. From 6 weeks to 6 months, a similar exercise program was followed, increasing functional activity and progressively working on calf muscle strength. Patients were discharged from physiotherapy when the contralateral limb range of motion level of the ankle and the pattern of gait were gained.

\section{Statistical analysis}

Statistical analyses were performed using NCSS 2007 software (Number Cruncher Statistical System, Kaysville, UT, USA). Data are reported as medians, and first and third quartiles. In comparisons of quantitative data among the three groups, the Kruskal-Wallis test was used, and to determine from which group the difference originated, the Mann-Whitney $U$-test with Bonferroni correction was applied. Values of $P<0.01$ and $P<0.05$ were considered to indicate statistical significance.

\section{Results}

The series initially included 24 Achilles tendons, of 19 males and five females, with a mean age of $42.00 \pm 11.85$ years. In the results of the gait analyses, no statistically significant difference was found among the three groups with regard to metatarsal pressure, first toe start, first toe finish, and heel start $(P>0.05)$. In addition, no statistically significant difference was found between the two treatment groups with regard to foot balance, percentage of heel contact, heel rotation, or walking phase change $(P>0.05)$. Nevertheless, a statistically significant difference was determined between the treatment groups and the control group $(P<0.001)$. The group comparisons are shown in Table 1.

There was no case of recurrent rupture or nerve injury. All patients were able to resume their prior activities after 6 months and regained normal ranges of motion, with a high rate of satisfaction. Most of the patients $(75 \%)$ were able to return to their previous level of activities. Results of gait analysis showed significant functional mobility deficiency during the swing phase, expressed as weakened active heel lift, mean peak heel pressure, metatarsal pressure, and foot balance.

\section{Discussion}

Acute Achilles tendon rupture management options include surgical and nonsurgical treatments. ${ }^{13}$ It is currently accepted that surgery should be performed in athletes and young patients, and that conservative treatment may be more appropriate for elderly patients. ${ }^{14}$ Previous trials have demonstrated that surgical treatments can reduce the risk of re-rupture

Table I Comparisons among groups

\begin{tabular}{|c|c|c|c|c|c|}
\hline & $\begin{array}{l}\text { Conservative } \\
\text { (with PRP) }(n=8)\end{array}$ & $\begin{array}{l}\text { Surgical } \\
(n=8)\end{array}$ & $\begin{array}{l}\text { Control } \\
(n=8)\end{array}$ & ${ }^{\text {a } P \text {-value }}$ & ${ }^{\text {bPost hoc }}$ \\
\hline & Median (QI, Q3) & Median (QI, Q3) & Median (QI, Q3) & & \\
\hline Age (years) & $43.5(37,52)$ & $39(35,4 I)$ & $47(30.5,51)$ & 0.525 & - \\
\hline Foot balance & $-3.5(-4,-3)$ & $-2.5(-3,-1.5)$ & $0(-2,0)$ & $<0.001 * *$ & $\mathrm{I}<3$ \\
\hline Metatarsal pressure $\left(\mathrm{N} / \mathrm{cm}^{2}\right)$ & $-16(-17,-16)$ & $-16(-17,-16)$ & $-17(-17,-16)$ & 0.626 & - \\
\hline Heel rotation & $12.5(11,13)$ & $12.5(12,13)$ & $5.5(4.5,6)$ & $<0.001 * *$ & $1,2>3$ \\
\hline Heel contact $(\%)$ & $53(49,58)$ & $51.5(45.5,62)$ & $36.5(31,43)$ & $0.007 * *$ & $1,2>3$ \\
\hline First toe start (ms) & $253.3(80.6,317.7)$ & $82.6(7.9,248.9)$ & $233.1(7.9,506.6)$ & 0.464 & - \\
\hline First toe finish (ms) & $757.4(705.3,84 I .6)$ & $746.7(638.8,805.4)$ & $675.7(648,702.3)$ & $0.090 *$ & - \\
\hline Heel start (ms) & $4(1.85,108.5)$ & $13.8(2.45,38.7)$ & $36.2(14.9,49.3)$ & 0.573 & - \\
\hline Heel finish (ms) & $470.7(433,568.1)$ & $377.7(237.2,532)$ & $246.7(225.7,306.4)$ & $0.007 * *$ & $1>3$ \\
\hline MPHP $\left(\mathrm{N} / \mathrm{cm}^{2}\right)$ & $1.9(1.65,2.1)$ & $2.2(1.7,2.4)$ & $0.4(0.2,0.5)$ & $<0.00 I^{* *}$ & $1,2>3$ \\
\hline
\end{tabular}

Notes: ${ }^{K}$ Kruskal-Wallis test; ${ }^{\circ}$ Mann-Whitney $U$-test (Bonferroni-corrected $P$-values are reported). QI: first quartile; $Q 3$ : third quartile. ${ }^{*} P<0.05$, ${ }^{*} * P<0.01$.

Abbreviations: PRP, platelet-rich plasma; MPHP, mean peak heel pressure. 
but may also cause a higher rate of postoperative complications, whereas conservative treatments are thought to have the advantage of a lower rate of complications, but with a higher risk of re-rupture. ${ }^{15}$ Thus, controversy regarding the optimal treatment method and postoperative rehabilitation continues. Despite the different concepts of treatment, the common goal is to achieve good and full functional capacity while avoiding re-rupture. ${ }^{16}$ Previous studies have tended to focus on re-rupture rather than functional results. ${ }^{8}$ In this study, surgical and conservative treatments were evaluated using a pedobarographic analysis.

This study aimed to quantify gait abnormalities after two different treatment modalities. The pedobarographic analysis confirmed that patients had a statistically significant deficit in MPFP in the first phase of rehabilitation. This points out weakness in the musculotendinous junction which is responsible for maintaining plantar flexion torque at the ankle joint. The muscle weakness and subsequent loss of balance within the gait cycle results in a less energy-efficient walking pattern. This is consistent with the limited walking distance and diffuse aching in the calf that affect many patients. The deficit in forefoot pressure is associated with a concomitant increase in the mean peak heel pressure on the affected side. This may describe the heel pain that also distresses patients, mostly in the first phase of rehabilitation. Many studies have compared different treatments for Achilles tendon rupture, focusing mainly on complications and results. Major complications secondary to surgical treatment of acute Achilles tendon rupture occur in up to $10 \%$ of cases and include deep infection, and tendon and skin necrosis with minor complications occurring in up to $15 \%$ of cases, commensurate with the development of skin problems. ${ }^{13}$ Rehabilitation after rupture of the Achilles tendon is slow and, for professional athletes, it can be a career-threatening injury. ${ }^{10}$ The average return to sport takes $\sim 6-9$ months. ${ }^{17}$ It has been reported that only $50 \%-60 \%$ of nonelite athletes regain their full pre-injury activity level. ${ }^{18}$ In the current study, all the patients were able to start their pre-injury activities after 6 months. Most of the patients $(75 \%)$ returned to their pre-injury level of sports activity. This may have been due to close monitoring at frequent intervals, and the efficacy of the rehabilitation program that was applied.

Another important problem after rupture of the Achilles tendon is gait abnormalities. ${ }^{10}$ Evaluation of such abnormalities is complex, although the initiation of novel techniques in pedobarography has simplified more precise analyses. ${ }^{10}$ In the current study, the two treatments were compared with each other and also with normal feet in the control group. In the results, no statistically significant difference was determined between the conservative (with PRP) group and the surgically treated group, but a statistically significant difference was found between both treatment groups and the controls. When the complications reported in the literature are taken into account, despite the low number of cases in the current study and the fact that there were no significant differences between the conservative (with PRP) group and surgery group, it indicates that, without surgery, the conservative treatment method can be used to avoid the risk of complications that may develop intraoperatively or postoperatively.

In recent years, the administration of PRP injections has become more common in orthopedic surgery and sports medicine practice. ${ }^{19,20}$ It may be difficult to convince patients to undergo conservative treatment using only a brace, when other patients undergo surgical repairs of their injured tendons at different medical centers. It is accepted that the currently available evidence does not fully support the efficacy of PRP administration. ${ }^{21}$ Thus, discussion of the supposed benefits of PRP injections, which are believed not to exceed the placebo effect was disapproved. ${ }^{22}$

The use of pedobarography for gait analysis was a major limitation of this study. Plantar pressures are only one aspect of gait analysis and additional studies are thus required to better understand the problems caused by rupture of the Achilles tendon. Nevertheless, the major functional deficit after such an injury lies within the musculotendinous junction of the region. Primary function of this unit is to maintain the plantar flexion torque at the ankle joint. Therefore, it is rational to initiate assessment of the causing plantar pressure deficiency. Future studies, ideally as part of randomized controlled trials, are essential to compare operative versus nonoperative treatments. Also, conservative treatment without PRP administration should be added as another study group.

\section{Conclusion}

The results of this study indicated that nonsurgically treated patients with Achilles tendon rupture showed signs of both anatomical and functional lengthening of the tendon. In our experience, conservative treatment provides satisfactory results in the management of acute ruptures of the Achilles tendon, regaining ankle mobility, gait strength, and stability. It was observed that surgically managed patients required longer rehabilitation phases to restore previous ankle mobility. No significant difference or complication was observed in the group treated conservatively versus the group treated surgically. Thus, conservative treatment 
choices should be an option except in the cases of active athletes. Future studies including 3D gait analyses and tendon biomechanical research would shed further light on the unanswered questions in this area.

\section{Disclosure}

The authors received no financial support and have no competing interests with regards to this paper.

\section{References}

1. Holm C, Kjaer M, Eliasson P. Achilles tendon rupture - treatment and complications: a systematic review. Scand J Med Sci Sports. 2015;25(1): e1-e10.

2. Wilkins R, Bisson LJ. Operative versus nonoperative management of acute Achilles tendon ruptures: a quantitative systematic review of randomized controlled trials. Am J Sports Med. 2012;40(9):2154-2160.

3. Cetti R, Christensen SE, Ejsted R, Jensen NM, Jorgensen U. Operative versus nonoperative treatment of Achilles tendon rupture. A prospective randomized study and review of the literature. Am J Sports Med. 1993; 21(6):791-799.

4. Nyyssonen T, Luthje P, Kroger $\mathrm{H}$. The increasing incidence and difference in sex distribution of Achilles tendon rupture in Finland in 1987-1999. Scand J Surg. 2008;97(3):272-275.

5. Clayton RA, Court-Brown CM. The epidemiology of musculoskeletal tendinous and ligamentous injuries. Injury. 2008;39(12):1338-1344.

6. Suchak AA, Bostick G, Reid D, Blitz S, Jomha N. The incidence of Achilles tendon ruptures in Edmonton, Canada. Foot Ankle Int. 2005; 26(11):932-936.

7. Cary DV. How to diagnose and manage an acute Achilles tendon rupture. JAAPA. 2009;22(8):39-43.

8. Keating JF, Will EM. Operative versus non-operative treatment of acute rupture of tendo Achillis: a prospective randomised evaluation of functional outcome. J Bone Joint Surg Br. 2011;93(8):1071-1078.

9. Jallageas R, Bordes J, Daviet JC, Mabit C, Coste C. Evaluation of surgical treatment for ruptured Achilles tendon in 31 athletes. Orthop Traumatol Surg Res. 2013;99(5):577-584.
10. Costa ML, Kay D, Donell ST. Gait abnormalities following rupture of the tendo Achillis: a pedobarographic assessment. J Bone Joint Surg Br. 2005;87(8):1085-1088.

11. Don R, Ranavolo A, Cacchio A, et al. Relationship between recovery of calf-muscle biomechanical properties and gait pattern following surgery for achilles tendon rupture. Clin Biomech (Bristol, Avon). 2007; 22(2):211-220.

12. Kawakami Y, Kanehisa H, Fukunaga T. The relationship between passive ankle plantar flexion joint torque and gastrocnemius muscle and achilles tendon stiffness: implications for flexibility. J Orthop Sports Phys Ther. 2008;38(5):269-276.

13. Soroceanu A, Sidhwa F, Aarabi S, Kaufman A, Glazebrook M. Surgical versus nonsurgical treatment of acute Achilles tendon rupture: a meta-analysis of randomized trials. J Bone Joint Surg Am. 2012;94(23): 2136-2143.

14. Raisbeck CC. Rupture of the Achilles tendon. J Bone Joint Surg Am. 2000;82-A(12):1804-1805.

15. Jiang N, Wang B, Chen A, Dong F, Yu B. Operative versus nonoperative treatment for acute Achilles tendon rupture: a meta-analysis based on current evidence. Int Orthop. 2012;36(4):765-773.

16. Follak N, Ganzer D, Merk H. The utility of gait analysis in the rehabilitation of patients after surgical treatment of Achilles tendon rupture. Eur J Orthop Surg Traumatol. 2002;12(2):90-95.

17. Myerson MS. Achilles tendon ruptures. Instr Course Lect. 1999;48: 219-230.

18. Leppilahti J, Orava S. Total Achilles tendon rupture. A review. Sports Med. 1998;25(2):79-100.

19. Foster TE, Puskas BL, Mandelbaum BR, Gerhardt MB, Rodeo SA. Platelet-rich plasma: from basic science to clinical applications. Am J Sports Med. 2009;37(11):2259-2272.

20. Mishra A, Harmon K, Woodall J, Vieira A. Sports medicine applications of platelet rich plasma. Curr Pharm Biotechnol. 2012;13(7): 1185-1195.

21. Middleton KK, Barro V, Muller B, Terada S, Fu FH. Evaluation of the effects of platelet-rich plasma (PRP) therapy involved in the healing of sports-related soft tissue injuries. Iowa Orthop J. 2012;32:150-163.

22. Filardo G, Kon E. PRP: Product Rich in Placebo? Knee Surg Sports Traumatol Arthrosc. Epub 2015 Sep 21.
Therapeutics and Clinical Risk Management

\section{Publish your work in this journal}

Therapeutics and Clinical Risk Management is an international, peerreviewed journal of clinical therapeutics and risk management, focusing on concise rapid reporting of clinical studies in all therapeutic areas, outcomes, safety, and programs for the effective, safe, and sustained use of medicines. This journal is indexed on PubMed Central, CAS,

\section{Dovepress}

EMBase, Scopus and the Elsevier Bibliographic databases. The manuscript management system is completely online and includes a very quick and fair peer-review system, which is all easy to use. Visit http://www.dovepress.com/testimonials.php to read real quotes from published authors. 